\title{
Numerical characterisation of the BSTMC: a reaction-to-fire test device for sandwich panel building systems
}

\author{
S. Sakji \& R. B. Martinez \\ Department of Safety, Structures and Fire, \\ Scientific and Technical Centre of Building Work (CSTB), France
}

\begin{abstract}
Composite roofs are widely used in the construction of buildings. They offer thermal and acoustical advantages of isolation. According to mandatory texts, their behavior under fire and their contribution to fire must be cheked and accepted before they can be used in construction. The ISO-13784-1 standard describes an experimental bench and a procedure for mesuring the contribution of horizontal elements. According to this standard, hot gas produced by the combustion in the upper side of the tested element and the gas coming from the room are melted together. It is not possible to distinguish between them and to analyse the gas produced in the test room which is the most relevant to take a decision about the people safety. In order to comply with this request, the CSTB has proposed and set on an innovative solution, BSTMC (Bench of Thermal Sollicitation and Caloremetric Measure) based on a minor modification of the ISO 13784-1 bench. This article aims at showing the part A of a work performed in order to characterize the BSTMC and to analyse the impact of the modification introduced. The first step consists of developing a numerical model of the BSTMC validated by experimental results. For this task, the Fire Dynamic Simulator (FDS) code is used. The second step consists of perfoming a parametric analysis combining different relevant parameters (design parameters, extraction power...).
\end{abstract}

Keywords: ISO 13784-1, sandwich panels, fire test, ISO 9705, FDS, CFD. 


\section{Introduction}

The characterisation of the reaction to fire of materials and systems is a major concern for the fire engineering and the performance based fire-engineering design. In the last twenty years, a mutation of fire engineering design has occurred, with a real conviction and will for switching from a prescriptive approach to a performance based approach. This is motivated mainly by $[1,2]$ (1) allocating resources in such a way to meet real needs (cost-effective design) instead of satisfying the redundancy arising from the prescriptive design, (2) promoting innovation by performing rational design for projects which can be considered unconventional to be covered by the prescriptive codes and (3) getting flexible design to satisfy safety criteria other than those prescribed by a given code.

The main difference between the prescriptive approach and the performance based design (PBD) is that, for the first, the fire resistance and reaction to fire of materials are prescribed by the mandatory texts in such a manner that the global objectives of security are assumed to be achieved. This approach is based mainly upon feedback and induces several redunduncies. For the PBD [3], the developpment of fire in buildings is determined precisely according to the actual calorific products and the caracteristics of the buildings. In this context, one of the most important input is the Heat release rate (HRR) of constitutive materials. Different test methods allow to get this parameter and to assess the fire behaviour of sandwich panels. The intermediate scale test method SBI (Small Burner Item) can be used to certify panels but different researches have demonstrated that on the one hand, the correlation between the SBI results and large scale test results is insufficient [4] and on the other hand, it is necessary that the tested panels reflect the end-use details of the whole structure which can not be met with the SBI equipement. It is clear that small scale testing is particularly interesting from the economical point of view on condition that their results are implemented in a manner compatible with the PBD. But, in some conditions it is difficult to catch and correctly take into account particular boundary conditions in modelling and there is a need to perform large scale tests in order to calibrate modelling and results of numerical simulations [5]. Nevertheless, the SBI results can be relevant when it comes to comparing different products and interesting for the developpment of innovative constructive solutions.

The ISO 13784-1 "Reaction-to-fire tests for sandwich panel building systems" gives a description of a large scale method for mesurement of the burning behaviour suitable to assess freestanding and self-supporting and frame supported sandwich panel [6]. The principle outputs are the HRR and the time to flashover. The facility is equipped with a standard room, an exhaust hood, a volumetric flow meter, and a $\mathrm{O}_{2} / \mathrm{CO}_{2} / \mathrm{CO}$ gas analyzer [7]. The standard room has dimensions of $3.6 \mathrm{~m}$ depth, $2.4 \mathrm{~m}$ width, and $2.4 \mathrm{~m}$ height, with a door of $0.8 \mathrm{~m}$ width and $2.0 \mathrm{~m}$ height; and its walls and ceiling are constructed from fireresistant materials with a thickness of $20 \mathrm{~mm}$ and a density of $500-800 \mathrm{~kg} / \mathrm{m}^{2}$. Some test series performed in European laboratories [4] revealed that the overall 
characterisation of products is related to joints and it was shown that the behaviour of joints and the possible ignition on the top of samples can be the major key factors causing the bad repeatability between the samples [8,9]. One way to circumvent this problem is to identify in the test method the quality of combustion gas released by joints.

For this matter and in order to improve the accuracy of the test method and the possibility of comparison between different products and also because it is important to identifiy the hazard coming from the inside of the room test, a modification of the configuration given in the ISO13784-1 has been performed and set on.

The goal of this work is to contribute to the characterisation of the BSTMC device and to estimate the impact of the new configuration on the general parameters influencing the combustion process of samples (temperature, gaz pressure, velocity...).

In this paper, the main results and conclusions about the impact of relevant parameters are exposed, namely the impact of the wind velocity, the impact of the extraction power and the impact of geometric parameters.

\section{Description of the set-up}

The BSTMC is a set based on a minor adjustment of the bench described in the ISO 13784-1. The modification proposed consists of separating the inner and the outer parts of the room test by adding an internal membrane separating the hood inner volume into two compartments (as shown in Figure 1), which gives a schematic representation of the used concept. The hot gases released in the inner and the outer sides of the tested specimen are extracted separately by means of two extractions grids connected to an axial fan by means of two pipes (cf. Figure 1). The HRR measure is also doubled. So, it is possible to get separate measures of the HRR, the first coming from the inside of the room and the second coming from the outer part of the product.

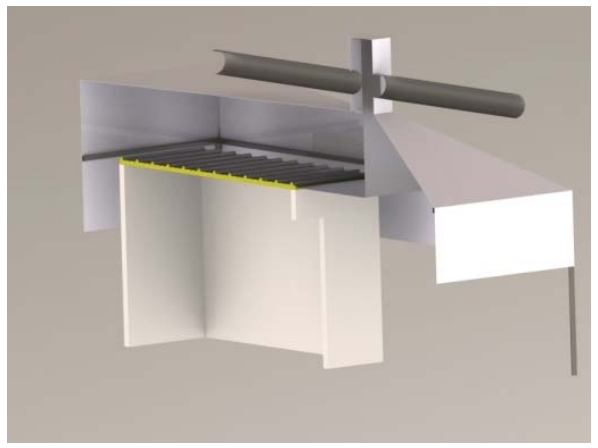

(a)

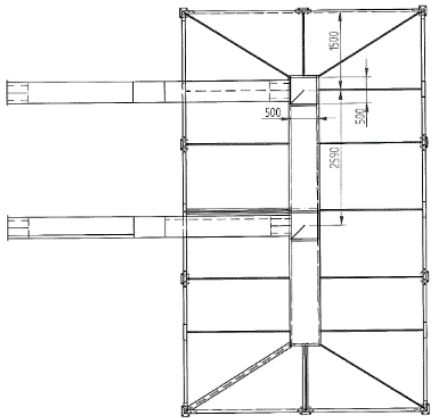

(b)

Figure 1: (a) Conceptual representation and (b) design plan of the BSTMC. 
It is possible, by modifying the configuration test, to comply with both standards, ISO 9705 [7] and ISO 13784-1 [1]. For the first case, linings can be fixed on the inner face of walls and the test can be then performed by analysing the HRR coming from the room test. For the second configuration, the roof complex is fixed on the walls of the room test and the measurement of HRR is performed using the two measurement lines. The power of sand gas burner is controlled using a mass flow meter in order to get $100 \mathrm{~kW}$ in the first 10 minutes of the test and $300 \mathrm{~kW}$ for the following 10 minutes. The extraction flow is controlled separately for the two lines.

It is to note that the system is conceived in such a way that the manipulation and installation of the specimen is made easy by taking off the hood (as shown in Figure 2).
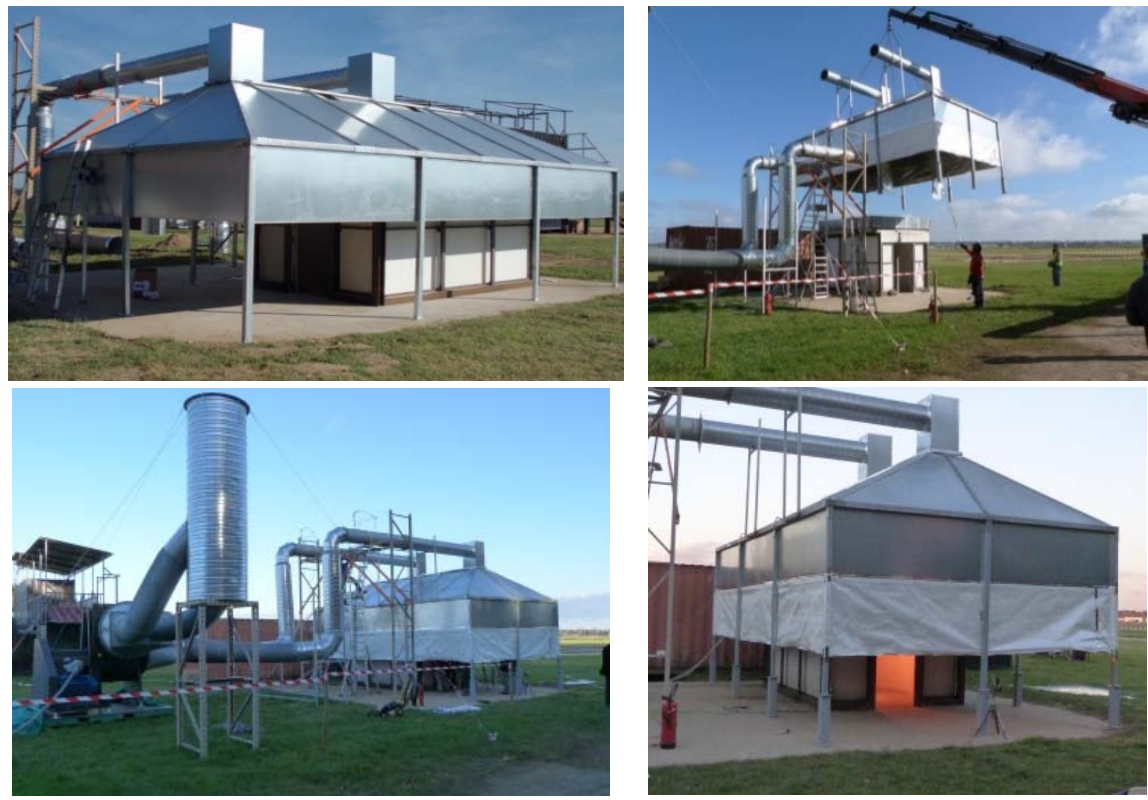

Figure 2: The BSTMC device proposed by the CSTB.

\section{Modelling}

This part is aimed at giving the influence of some relevant parameters on the internal temperature and gas flows which determine the combustion of sandwich panels and then the measured HRR. The chosen parameters are the wind intensity, its direction, the extraction flow, and the dimensions of the lower part of the enclosure. In all numerical simulations, the gas burner power as a function of time is the same as that given by the ISO 13784-1. 


\subsection{Numerical tool}

The numerical tool used in this work, is the version 5.5 of FDS [10] code (Fire Dynamics Simulator) developed by the National Institute of Standards and Technology (NIST). FDS has been conceived in order to solve the main physical phenomena governing the fires (combustion in diffusion regime, smoke dynamics and radiation of heat phenomena). FDS solves the low Mach (incompressible fluids, $\mathrm{Ma}<0.3$ ) form of the conservation equations of mass, quantity of movement and energy. The turbulence is solved using the LES (Large Eddy Simulation) approximation and the combustion is solved using an infinite rate reaction approximation. The partial derivatives of the conservation equations are approximated using an explicit finite difference schema, updated in time on a three-dimensional rectilinear grid.

\subsection{The BSTMC geometry}

The Figure 3 gives two views of the BSTMC numerical model used in the parametric analysis. It is formed by a rectangular room and a calorimetric hood, the membrane allowing to separate the hot gas coming from the specimen and the hot gas generated inside the room is in green. In order to reduce the grid number, and save CPU time, we do not model the exhaust pipes (cf. Figure 3) of the real device and we apply directly the extraction condition on the extraction grids (cf. blue squares at the top of the calorimeter hood).

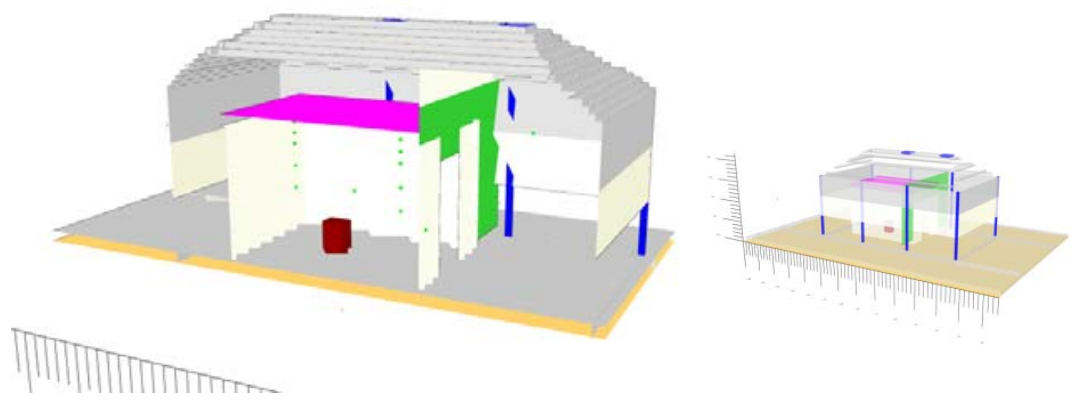

Figure 3: The BSTMC internal configuration.

The dimensions of the meshed domain, conceived to be solved in a parallel way, are $9.5 \mathrm{~m}$ according to $\mathrm{x}$ axis, $15.2 \mathrm{~m}$ according to $y$ axis and $3.2 \mathrm{~m}$ according to the $\mathrm{z}$ axis. These dimensions were chosen after a mesh optimization process in order to get a reasonable simulation time. The domain mesh retained was built and formed by six different meshes (cf. Figure 4). The calculations use six processors under the MPI parallel protocol. The total number of grids of the six meshes was about 455480 grids; the average volume per cell was around of $0,001 \mathrm{~m}^{3}$. The average CPU time for each simulation was around of 130 hours by 60 minutes of physical time. 


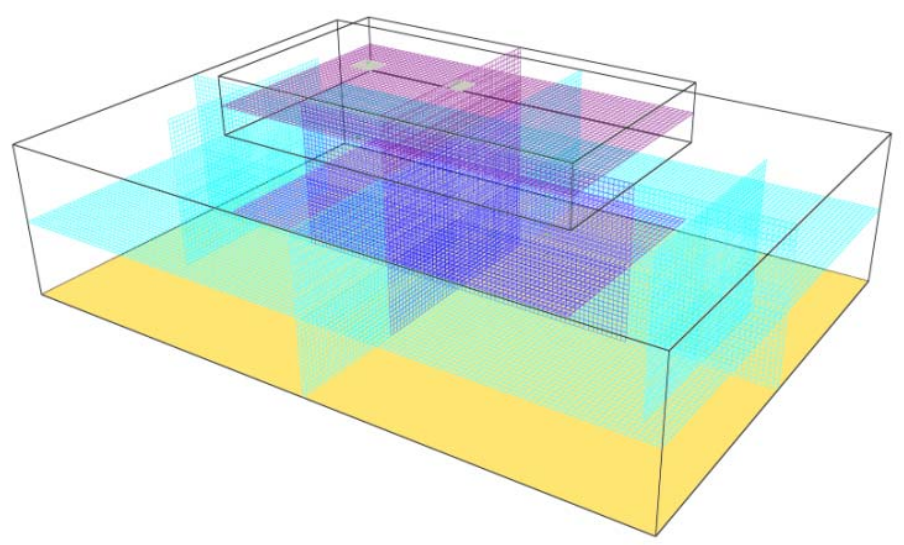

Figure 4: The BSTMC numerical mesh.

\section{Results and discussion}

\subsection{Wind speed influence}

Since the BSTMC is an outdoor setup, it is necessary to verify that the ambient conditions comply with the recommendation of the ISO 13784-1. In fact, according to the former standard, the horizontal wind speed measured at a horizontal distance of $1 \mathrm{~m}$ from the doorway shall not exceed $1.75 \mathrm{~m} / \mathrm{s}$. For this matter, several numerical simulations have been performed in order to define conditions of use of the BSTMC. Table 1 gives the velocity of particles at the prescribed distance as a function of different wind speeds and it appears that the prescribed recommendation can be verified only if the wind speed is less than $16 \mathrm{~km} / \mathrm{h}$. This value can be used in order to verify if the climate conditions are acceptable or no to perform test in a planned date.

Table 1: Velocity of particles in front of the doorway for different values of wind speed.

\begin{tabular}{|c|c|}
\hline $\begin{array}{c}\text { External wind } \\
\text { speed } \\
(\mathrm{km} / \mathrm{h})\end{array}$ & $\begin{array}{c}\text { Wind speed in front of the } \\
\text { room test door }(\mathrm{m} / \mathrm{s})\end{array}$ \\
\hline 5 & 0.51 \\
\hline 10 & 1.09 \\
\hline 15 & 1.55 \\
\hline 16 & 1.67 \\
\hline 17 & 1.81 \\
\hline 18 & 1.95 \\
\hline 19 & 2.05 \\
\hline
\end{tabular}




\subsection{Influence of extraction power}

Several numerical simulations have been performed combining different extraction powers in the two lines of extraction in order to get the influence of this parameter on the temperature inside the room test and especially the thermal action which a tested sandwich panel would be subjected to. The set $\left\{2 \mathrm{~m}^{3} / \mathrm{s}\right.$, $\left.2.5 \mathrm{~m}^{3} / \mathrm{s}, 3 \mathrm{~m}^{3} / \mathrm{s}\right\}$ of extraction flows has been combined and nine numerical simulations have been performed. Temperatures in the room test have been extracted for two positions and two heights (four thermocouples TC1, TC5, TC6 and TC10). The thermocouples $\mathrm{TC} 1$ and $\mathrm{TC} 5$ are at $50 \mathrm{~cm}$ from the burner, thermocouples TC6 and TC10 are at $1 \mathrm{~m}$ in the front left corner of the room. The thermocouples TC1 and TC6 are at $20 \mathrm{~cm}$ from the roof. Finally, TC5 and TC10 are at $1.4 \mathrm{~m}$ from the roof.

From Figures 5 and 6 which correspond to some results of the numerical simulations, some remarks can be drawn:

1) the influence of modifying the extraction flow is not significant when the burner power is $100 \mathrm{~kW}$;

2) The maximum difference of temperature is about $50^{\circ} \mathrm{C}$ when the power of the burner is $300 \mathrm{~kW}$;

3) There is no significant difference in the temperature when the extraction flow is $2.5 \mathrm{~m}^{3} / \mathrm{s}$ or $3 \mathrm{~m}^{3} / \mathrm{s}$ (same flow in the both lines);

4) When the extraction flow is higher in the rear line, the thermal action is $50^{\circ} \mathrm{C}$ higher.

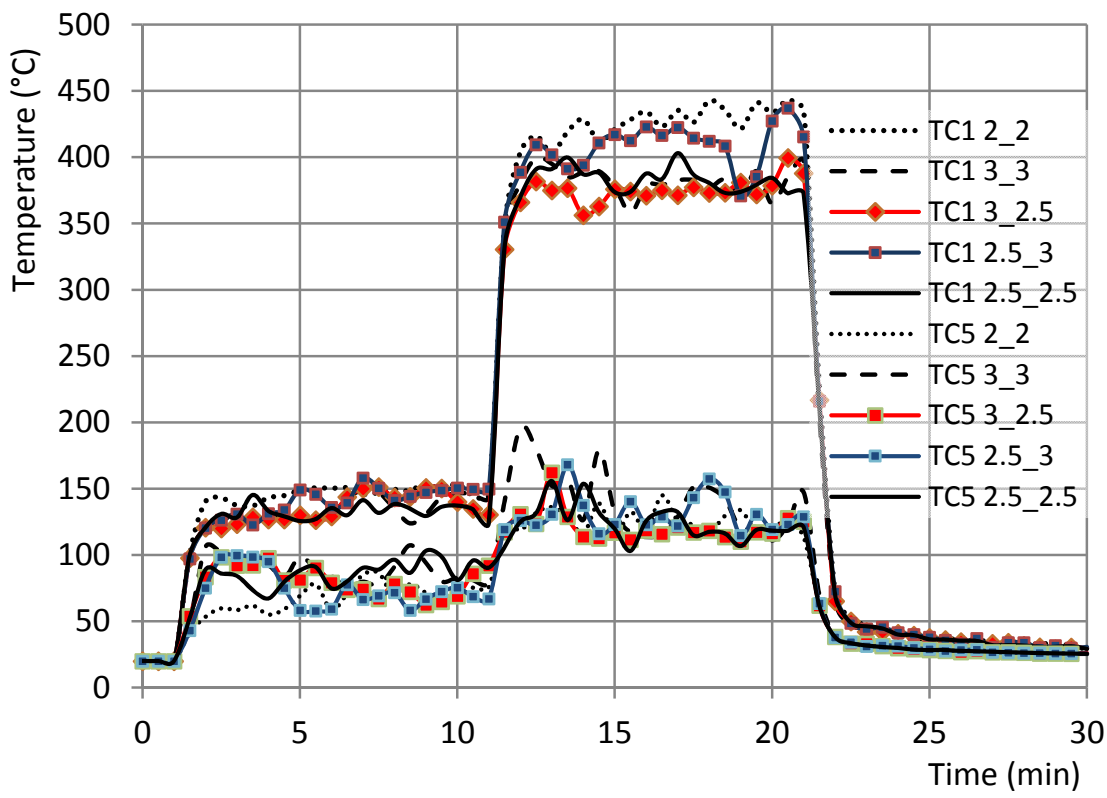

Figure 5: Temperature as a function of time given for the thermocouples TC1 and TC5 according to a different combination of flow extraction into the two lines (flow in the front line _flow in the rear line). 


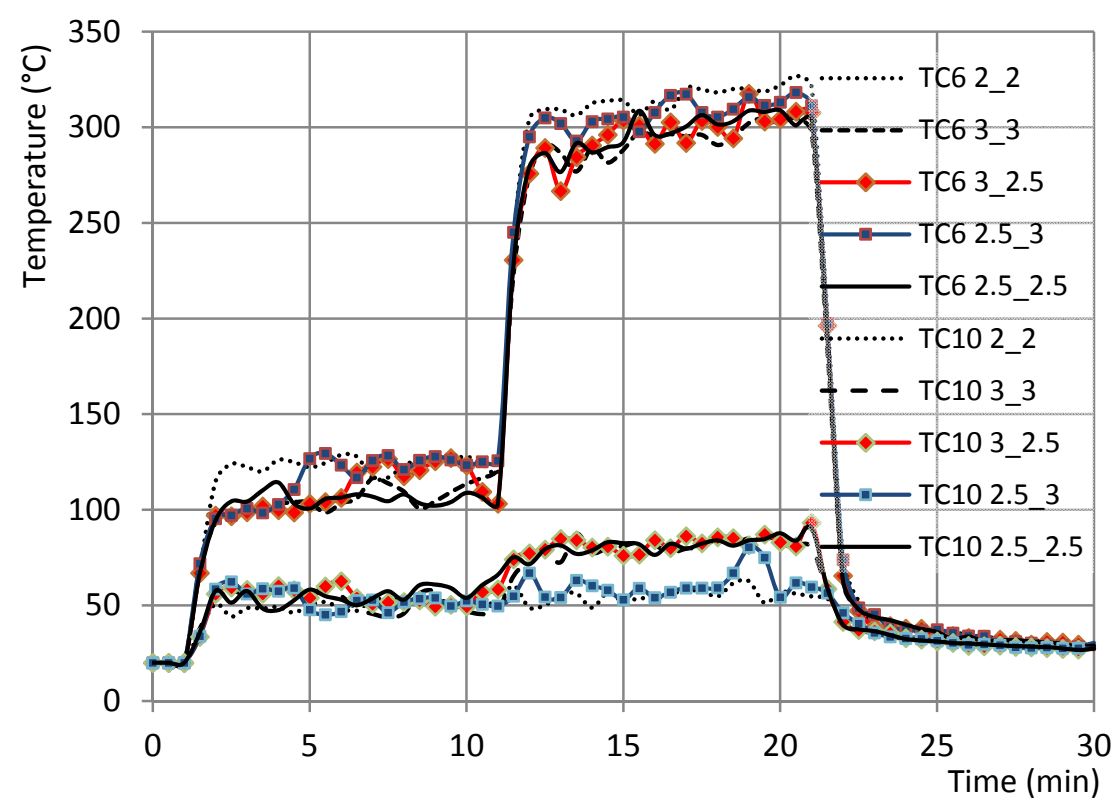

Figure 6: Temperature as a function of time given for the thermocouples TC6 and TC10 according to a different combination of flow extraction into the two lines (flow in the front line _ flow in the rear line).

\subsection{Geometric parameter}

During tests some leaking smoke was observed below the vertical membrane of the hood and it was decided to increase its size in order not to disturb the HRR measure. In order to get the influence of this parameter on the temperature inside the room test and to justify the modification done, numerical simulations were performed for three dimensions (size 1: the actual dimension, size 2: the actual dimension $+0.4 \mathrm{~m}$ and size 3 : the actual dimension $-0.4 \mathrm{~m}$ ). Figure 7 gives the temperature as a function of time for the three mentioned configurations and at different height in the room test $(20 \mathrm{~cm}$ from the roof for $\mathrm{TC} 1,60 \mathrm{~cm}$ from the roof for TC3 and $140 \mathrm{~cm}$ from the roof for TC5). From these results one can conclude that this parameter has not a significant influence on the thermal action. Nevertheless, results of numerical simulation (cf. Figure 8) show that for the size 3 configuration there is a smoke leak under the hood. This phenomenon was not observed for the two other configurations. In order to reduce errors of HRR measure, it is important to perform tests with at minimum the size 1 for this parameter. 


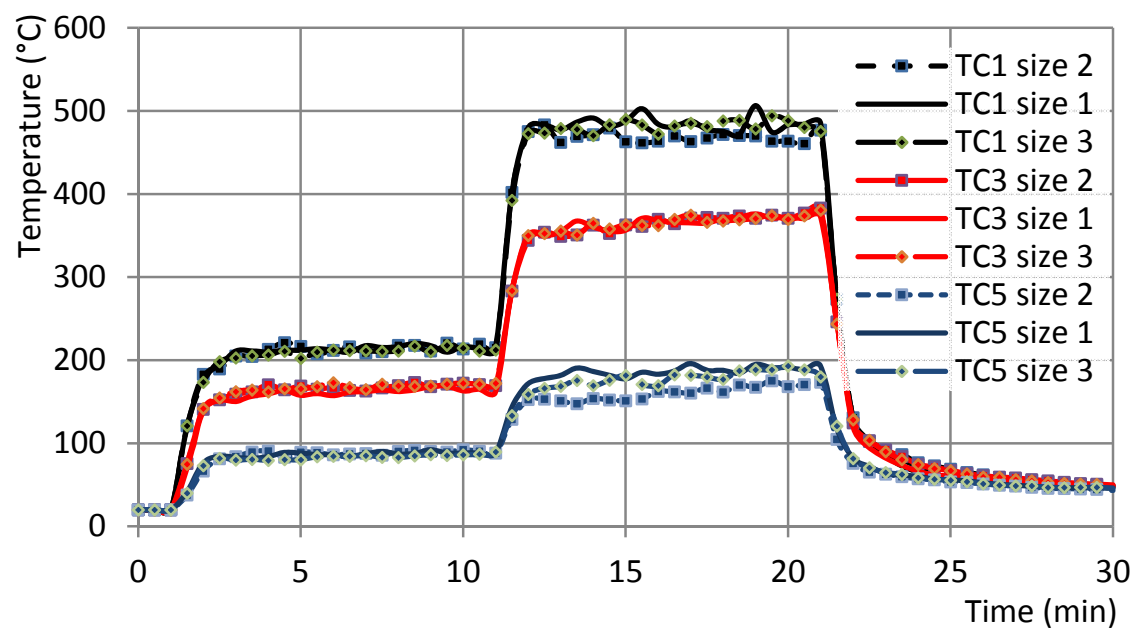

Figure 7: Temperature as a function of time for different dimensions of the vertical membrane of the hood. TC1 gives the temperature at 20 $\mathrm{cm}$ from the roof, TC3 gives the temperature at $60 \mathrm{~cm}$ from the roof and TC5 gives the temperature at $140 \mathrm{~cm}$ from the roof.

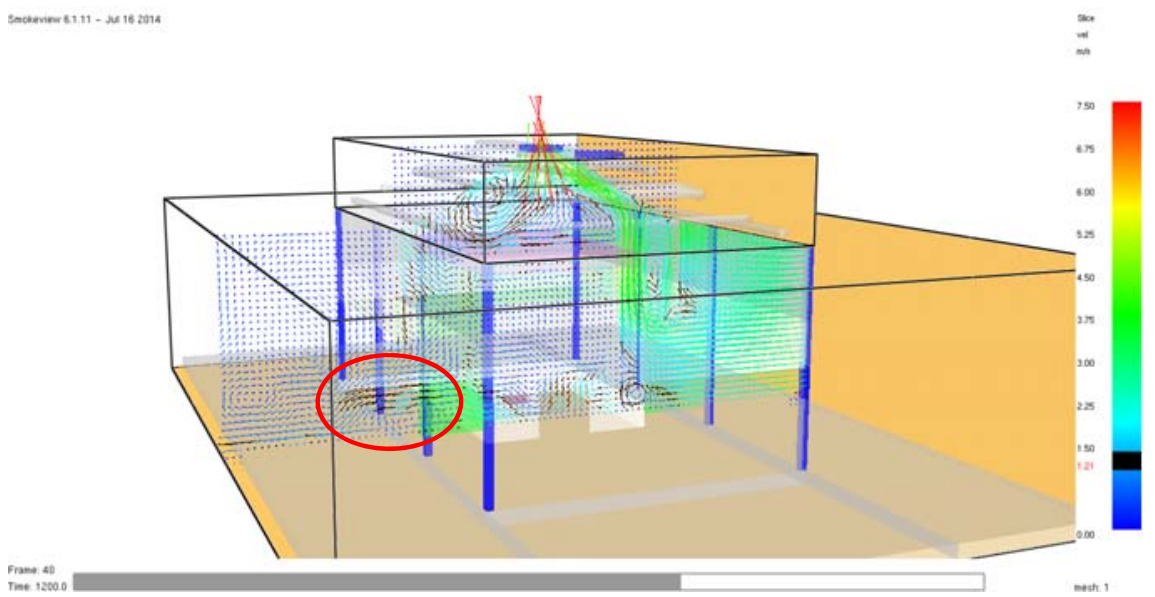

Figure 8: Slice of velocity vector at 20 minutes of numerical simulation showing the smoke leak under the hood membrane.

\section{Conclusion and perspectives}

The BSTMC is a set up solution allowing to perform tests according to both ISO9705 and ISO13784-1 standards which gives the possibility to ameliorate the accuracy of test results by identifying the contribution of joints in the combustion process. Numerical simulation based on FDS modelling has been 
performed in order to define relevant parameters and their boundaries in order to comply with these standard prescriptions.

In this first numerical work, we simulate only the combustion process of the gas burner. Further development will include the combustion and the heat released by the studied specimen in order to simulate the combustion of sandwich panels. A probabilistic modelling is also in progress in order to take into account uncertainty coming from errors of measure.

\section{Acknowledgements}

The conception and the implementation of the BSTMC would not have been possible without the effective contribution of D. Pardon and G. Mitanchez (VI incendie). The authors would like to thank Q. Jullien for his implication in this work.

\section{References}

[1] V. Babrauskas, Performance-Based building codes: What will happen to the levels of safety? Fire science and Technology Inc., 9000-300th Place SE, Issaquah WA 98027, USA.

[2] V. Beck, Performance-based Fire Engineering Design and its Application in Australia, Fire Safety Science - Proceeding of the Fifth International Symposium, 23-40.

[3] Paul A. Croce et al., The International FORUM of Fire Research Directors, A position paper on performance-based design for fire code applications. Fire Safety Journal 43 (2008), 234-236.

[4] J. Axelsson and P. Van Hees, New data for sandwich panels on the correlation between the SBI test method and the room corner reference scenario. Fire and Materials 29 (2005), 53-59.

[5] C. Wade, Future directions in fire testing of building products, Building for a Global Future - Australia's Built Environment, September 2003.

[6] International Organization for Standardization, Reaction-to-fire tests for sandwich panel building systems. Part 1: Test method for small rooms, Designation ISO 13784-1. December 2002.

[7] International Organization for Standardization, Fire tests. Full-scale Room Test for Surface Products, Designation ISO 9705: 1993(E), American National Standards Institute, Boston, MA, USA, 1993.

[8] P. Van Hees \& P. Blomqvist, A summary of fire regulations, requirements and test methods for technical textiles used in buildings, Fire Technology, SP Report 2007:20.

[9] P. Johansson and P. Van Hees, Development of a test Procedure for Sandwich Panels using ISO 9705 Philosophy, Nordest Project nr 1432-99, SP Report 2000:26.

[10] K. B. McGrattan et al., NIST special publication 1018 Fire Dynamics Simulator Technical Reference Guide, NIST, 2012. 